\title{
moBeat: Using Interactive Music to Guide and Motivate Users During Aerobic Exercising
}

\author{
Bram van der Vlist • Christoph Bartneck • \\ Sebastian Mäueler
}

Published online: 5 March 2011

(c) The Author(s) 2011. This article is published with open access at Springerlink.com

\begin{abstract}
An increasing number of people are having trouble staying fit and maintaining a healthy bodyweight because of lack of physical activity. Getting people to exercise is crucial. However, many struggle with developing healthy exercising habits, due to hurdles like having to leave the house and the boring character of endurance exercising. In this paper, we report on a design project that explores the use of audio to motivate and provide feedback and guidance during exercising in a home environment. We developed moBeat, a system that provides intensity-based coaching while exercising, giving real-time feedback on training pace and intensity by means of interactive music. We conducted a within-subject comparison between our moBeat system and a commercially available heart rate watch. With moBeat, we achieved a comparable success rate: our system has a significant, positive influence on intrinsic motivation and attentional focus, but we did not see significant differences with regard to either perceived exertion or effectiveness. Although promising, future research is needed.
\end{abstract}

Keywords Sonification - Auditory feedback - Endurance exercising $\cdot$ Exercise coach $\cdot$ Interactive music

B. van der Vlist $(\bowtie) \cdot$ C. Bartneck

Department of Industrial Design, Eindhoven University

of Technology, Den Dolech 2, 5600 MB Eindhoven,

The Netherlands

e-mail: b.j.j.v.d.vlist@tue.nl

S. Mäueler

Department of Care and Health Applications, Philips Research

Eindhoven, Eindhoven, The Netherlands
With a growing part of the developed countries' population having a sedentary lifestyle, overweight is an increasing problem. People suffer from related diseases, such as type 2 diabetes, cardiovascular diseases and cancer. During everyday life, many people are not physically active enough to stay fit and maintain a healthy body weight. Therefore, encouraging people to exercise is increasingly important. Fitness centres are gaining in popularity, as are exertion games like Nintendo Wii and Wii Fit (2008). The consumer is becoming more conscious of the importance of a healthy and balanced lifestyle. The food industry is offering an increasing number of diet products and detailed product information to enable the consumer to eat healthy. Governments and health organizations (Stichting Voedingscentrum Nederland, the Netherlands) are advocating a healthy lifestyle with expressions such as: "move at least 30 min a day", "be aware of what you eat, women need 2,000 kcal and men 2,500 kcal a day".

Products like Nike + iPod (2008), Samsung and Adidas' miCoach (2008) and Yamaha's BODiBEAT (2008) are emerging to make aerobic exercising, running in particular, more easy and enjoyable. These systems are essentially MP3 players with some additional sensors. More traditional training aids, such as heart rate (HR) monitors in the shape of wrist-top computers (Polar Electro 2008; Suunto 2008) are becoming more advanced, with the incorporation of step counters and GPS modules. Although these HR monitors are ideal for guiding more advanced training programs, they often lack true motivational components. Fitness MP3 players, on the other hand, address motivation but lack the precise and dedicated feedback and functions that traditional heart rate monitors offer.

In this paper, we present an exploration of options for providing auditory feedback in an intuitive and motivating way to guide and enhance training performance. Before we 
arrive at our design proposal, we present background information and theory. We conclude our paper with a benchmark of our design against existing, competing products in terms of intrinsic motivation, attentional focus, and rate of perceived exertion.

\section{Background}

\section{Exercise Physiology}

We restrict our focus to cardio respiratory (CR) fitness because this is considered to provide a good measure of a person's bodily fitness; it has many important benefits, as we will describe later on.

When executed regularly, endurance exercises (also called aerobic exercises) can lead to increased CR fitness. In aerobic exercises aerobic metabolism (in contrast with anaerobic metabolism) is the main supplier of energy. Aerobic exercises can have a relatively long duration, employ large muscle groups, and are rhythmic or dynamic in nature. Intensity and duration are important measures of aerobic exercising. Among the recognized benefits of doing regular aerobic exercises are: strengthening the muscles involved in respiration; strengthening and enlarging the heart muscle, improving its pumping efficiency and reduce the resting heart rate and exercise heart rate at a certain load (due to increased efficiency); toning muscles throughout the body; improving blood circulation efficiency, reducing blood pressure; increasing the total number of red blood cells in the body, facilitating transport of oxygen; improving mental health (American College of Sports Medicine [ACSM] 2006).

Physical activity and aerobic exercises can reduce the occurrence of cardiac events, hypertension, type 2 diabetes mellitus, several types of cancer, osteoporosis, gallbladder disease, obesity, depression, anxiety, and the risk of stroke, as well as improve life expectancy (ACSM 2006). There are also numerous performance benefits and benefits in daily life functioning.

For different fitness goals, different types of exercises are needed. To assure effective aerobic exercising, exercise physiology offers ways to perform tests (exercise testing) to obtain data about the current level of fitness that can be used as a starting point for exercising. There are several strategies to obtain this, ranging from clinical exercise testing to simple, sub-maximal tests that are suitable for the home environment. Step tests like the Canadian Home Fitness Test (Jette et al. 1976) can be performed easily and deliver satisfactory results that can be used to set fitness goals and establish individual exercise sessions. A welldefined exercise session includes a warming-up, stimulus or condition phase, possibly recreational activities and a cooling-down phase. The stimulus or conditioning phase incorporates the actual exercise, in our case endurance exercises. Endurance exercises should be performed at the correct intensity, depending on the person's level of fitness and personal goals. Intensity-based endurance exercising by means of target heart rate (intensity) zones is one of the most suitable ways to guide endurance exercises. Training heart rate expressed in a percentage of $\mathrm{HR}_{\max }$ (maximum heart rate) or HRR (Heart Rate Reserve) can be used as a measure of intensity.

\section{Auditory Feedback in Exercising}

The use of audio, music and auditory feedback in a sports context is not new. BODiBEAT (Yamaha) and Philips' IM4Sports (Wijnalda et al. 2005) are examples of advanced MP3 players that are capable of selecting music with a pace matching the heart rate or running pace. In the case of IM4Sports, even the tempo of the music is altered - up to a certain limit-for more accurate matching.

The aim of these devices is to support and guide users' training effort and intensity throughout a training session. Nike and Apple (Nike + iPod) launched extensions to existing MP3 players that allow these players to store training data obtained by adding a stride sensor; one that monitors running pace, stride length and overall distance. The results can be reviewed after the training session on a personal computer. During the exercise, one can listen to one's favourite music and if a motivational impulse is needed, the iPod can play a pre-programmed 'power song'. Music mixes, specifically designed to support running, can be downloaded to ensure 'runnable' music. Adidas and Samsung take it a step further with miCoach as they also incorporate a heart rate belt connected to a specially designed fitness phone. Besides helping with setting up a training schedule and providing the user with music during the exercise, it can also select songs that are motivating when you need to speed up or give real-time spoken feedback on the covered distance, time and heart rate.

Polar, Suunto and others have developed advanced training packages that include multiple sensors (like GPS, heart rate, stride, cadence, speed, etc.). Sensor data are collected and presented through an LCD screen on a wristtop (wristwatch-like) computer. Auditory feedback can be used as an option, to mark pre-set limits to guide training intensity, and is given in the form of watch-beep like tones (that are often regarded as annoying). As opposed to exercise MP3 players and phones, these heart rate monitors allow for precise guiding using warming-up, cooling-down and dedicated intensity zones.

Looking at the growing number of existing products, there is still a gap between fitness MP3 players and heart 
rate monitors. Fitness MP3 players are popular lifestyle products that might enhance motivation, but often fail in substituting as real fitness coaches. Heart rate monitors have scientific backing, and could serve as advanced personal training computers, however, they do not address motivation and are merely targeted at exercise enthusiasts. An opportunity exists to combine the motivational features of fitness MP3 players with the advanced functionalities of heart rate monitors into a design that offers intuitive use and advanced training functionality backed with research and sports medical knowledge in a motivating fashion. Music can be used for motivation and guidance, given it is known for its influence on rhythmic movement (Karageorghis et al. 1999; Karageorghis and Terry 1997). Using physiological data to generate musical events is therefore an interesting way of giving people feedback and guidance, without being annoying.

\section{Motivation Through Music}

Motivation plays an important role in endurance exercising (Ryan et al. 1997). People usually perceive exercising as boring and unpleasant: a certain amount of motivation is needed to complete a training session. Motivation becomes more important if exercising needs to be maintained over a longer period of time. Intrinsic motivation is the type of motivation that occurs when people engage in an activity without having external pressure or influences. Interest and enjoyment play an important role in such an activity. Factors that are known to influence intrinsic motivation are: the interest and enjoyment a person has with the activity, the degree in which they are able to control the activity (for instance the amount of effort they put in it), the degree in which they succeed in the activity, and the relevance or value the activity has for them (Ryan et al. 1997; Deci and Ryan 1985).

Research has indicated that music has a beneficial effect in a sports and exercise context. Music hasamongst other effects-the capacity to capture attention, increase work output and encourage rhythmic movement. These effects all have a potential application in the sports domain (Terry and Karageorghis 2006). In their original conceptual framework for predicting psychophysical effects of music, Karageorghis et al. (1999) mention four important motivational qualities of music: rhythm response, musicality, cultural impact and association. These four factors are hierarchical with rhythm response as the most important. In another framework in a sports context, Karageorghis and Terry (2001) state that the main benefits for athletes would be: “(a) increased positive moods and reduced negative moods; (b) pre-event activation or relaxation; (c) dissociation from unpleasant feelings such as pain and fatigue; (d) reduced ratings of perceived exertion (RPE) especially during aerobic training; (e) extended work output through synchronization of music with movement; (f) enhanced acquisition of motor skills when rhythm or association is matched with required movement patterns; ( $\mathrm{g}$ ) increased likelihood of athletes achieving flow states; and (h) enhanced performance via the above mechanisms." (Terry and Karageorghis 2006, p. 415) Besides these benefits Szabo et al. (2001) found that the effect of moving from a slow musical tempo (in BPM) to a higher musical tempo produces an ergogenic effect.

Another relevant psychological mechanism is attentional focus. Dissociation, or purposeful focusing away from unpleasant feelings of pain and fatigue (that are often related to endurance exercising), is a frequently used strategy in exercising. External stimuli such as music and virtual reality applications (IJsselsteijn et al. 2004) are designed to lead the focus away from internal body sensations during exercise, causing less focus on distress cues and possibly leading to a lower rate of perceived exertion (RPE). Research has shown that amateur sportsmen benefit from dissociative strategies, while sports enthusiasts and competitive sportsmen benefit more from associative strategies (Brewer et al. 1996). We expect that the use of music during exercising has positive influence on (intrinsic) motivation. Additionally, music is expected to provide an external focus of attention, distracting the focus away from unpleasant sensations associated with endurance exercising.

\section{Design}

We named our design concept moBeat, which is a contraction of the first two letters of motivation and movement/move/motion, and the word beat, which refers to heartbeat and the beat of music. MoBeat provides users with interactive music during exercising. This music guides users to train in the correct intensities to optimize training performance. It provides feedback on performance by means of rewards. The music tempo adapts to the users' training pace, informing them to speed up or slow down when going too slow or too fast, and rewards users with richer music when training at the correct intensity.

Our design aims at persons who need motivation to succeed in maintaining the habit of endurance exercising, and who are willing to exercise in a healthy and effective way. This motivation may help them to overcome the boring character of most endurance sports and to distract them from negative physical sensations. Also, moBeat is suited for exercising at home. In the following section we 
describe the system architecture and functional model of the prototype that was developed and tested.

\section{System Architecture}

We designed and developed a research prototype for use on a stationary bicycle. It consists of hardware, software and MIDI music. Below we give a description of the components in each category.

\section{Hardware}

To obtain cycling (rear wheel) speed and cycle cadence (both measured in rotations per minute), we used a commercially available cadence sensor and speed sensor. These sensors, normally attached to cycling computers, are essentially reed switches (electric switches operated by applying a magnetic field) that are closed by a magnet affixed to a spoke in the wheel and one of the crank arms.

To obtain heart rate, a commercially available heart rate belt is used (a Polar T61 coded heart rate belt), because these belts are designed to be used with HR monitors during exercising. The HR belt is connected to a receiver (Polar RMCM-01) that is specifically designed to read Polar HR belts.

We used a circuit board built by SparkFun (Julio 2008) to read the sensor values. It contains: the Polar receiver, a microprocessor with an algorithm to calculate heart rate values, a prototype area with several digital and analogue I/O pins, and a serial-over-USB communication interface. The cadence and speed sensors are attached to two digital inputs. Values are read by the software through a simple serial master/slave interface. For more information and a circuit drawing of the hardware see (Julio 2008). Figure 1 shows an overview of the system hardware. Figure 2 depicts the position of the sensors and hardware on the bicycle.

\section{Software}

In the current prototype the software is written in Max/ MSP (Cycling '74 2008), a graphical programming environment that is well suited to manipulate (MIDI) audio. The software handles the serial communication and takes care of the main functionality of the system.

In the current prototype, we use music files in MIDI format that allows for easy control of musical richness and music tempo. Raw MIDI data (a series of MIDI events) is generated by a MIDI sequencer (currently a MIDI file running in Apple's Logic Pro) and is sent on separate MIDI channels over a virtual MIDI port to our software. The control software passes-on or blocks certain MIDI channels, controlling the musical richness and audio cues. The software sends out the processed MIDI data in a similar fashion as mentioned above to Logic Pro (Apple Inc. 2008). Software instruments in Logic Pro translate the processed MIDI data into audible sound. Figure 3 shows an overview of the software architecture.

\section{Functional Model}

MoBeat has three pivotal features that lead to the desired effect:

1. To make sure that the music is always in sync with the training pace (frequency of the repetitive movements that are the basics of the exercise), the music pace expressed in beats per minute (BPM) is defined by the training pace. In case of cycling the BPM of the music will be the cycling cadence (in RPM) multiplied by two.

2. Feedback on compliance to the prescribed training intensity - the training HR being in or outside the HR zone prescribed by the training program-is provided in the form of musical richness. Layers of similar musical features define musical richness. In the current
Fig. 1 Schematic overview of the system hardware

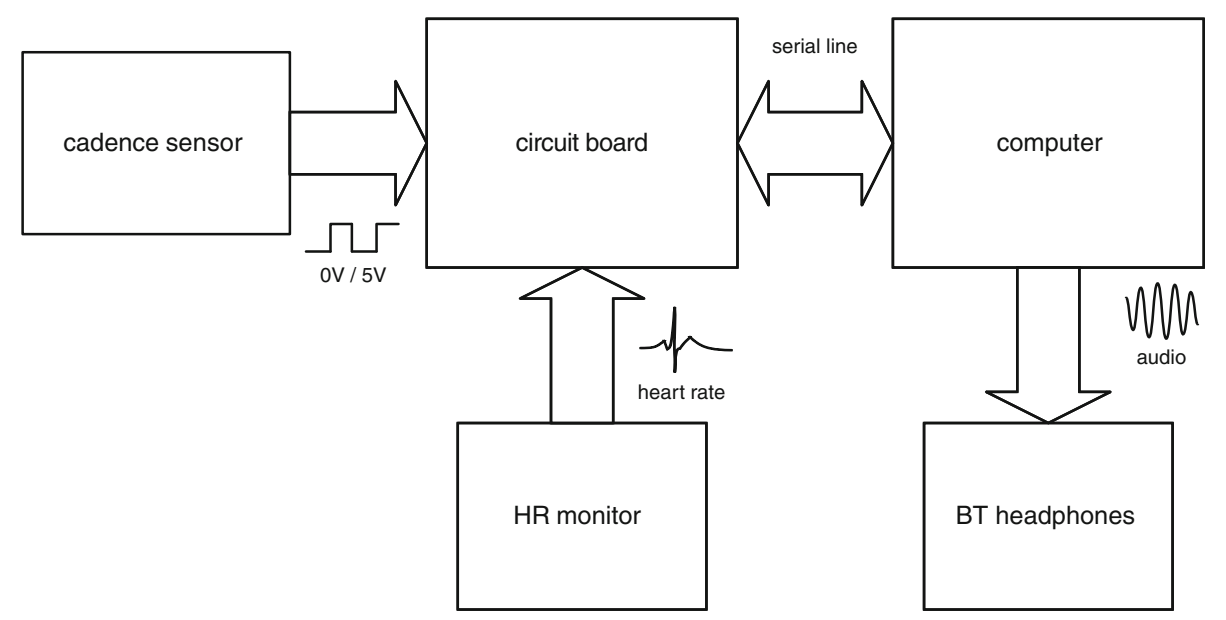


Fig. 2 The position of the sensors on the bike

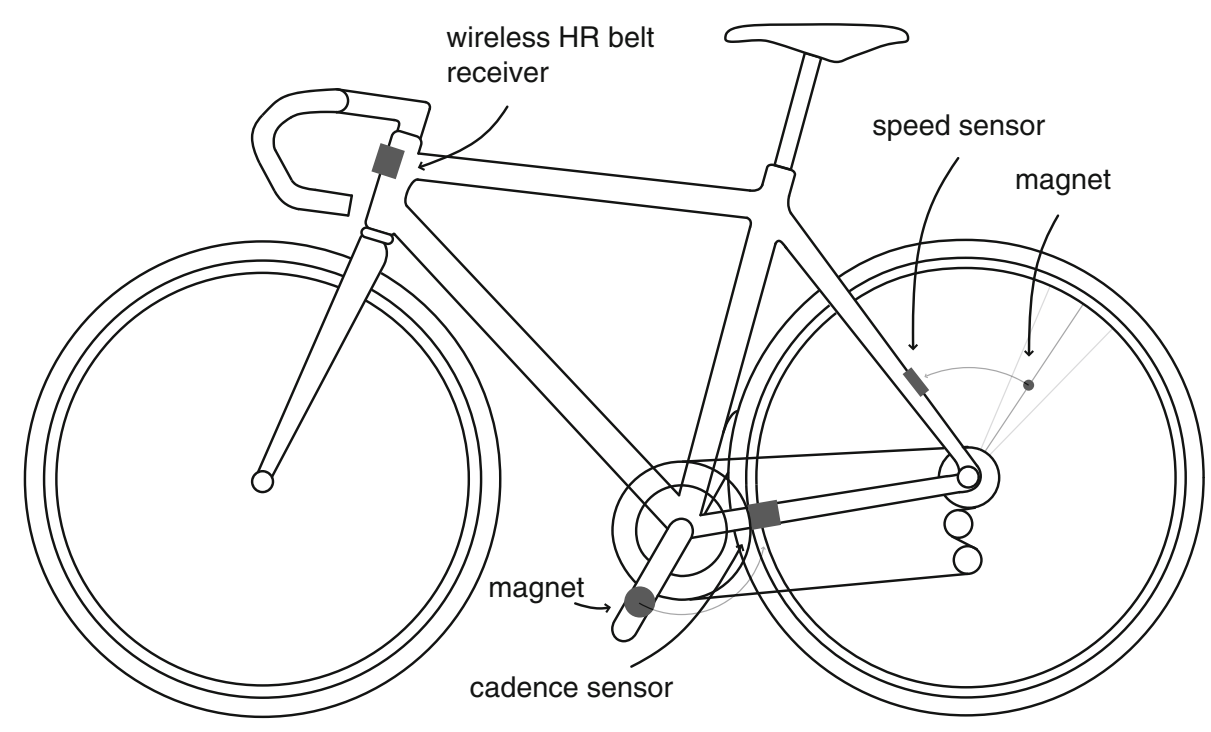

Fig. 3 Overview of the software running on the $\mathrm{PC}$

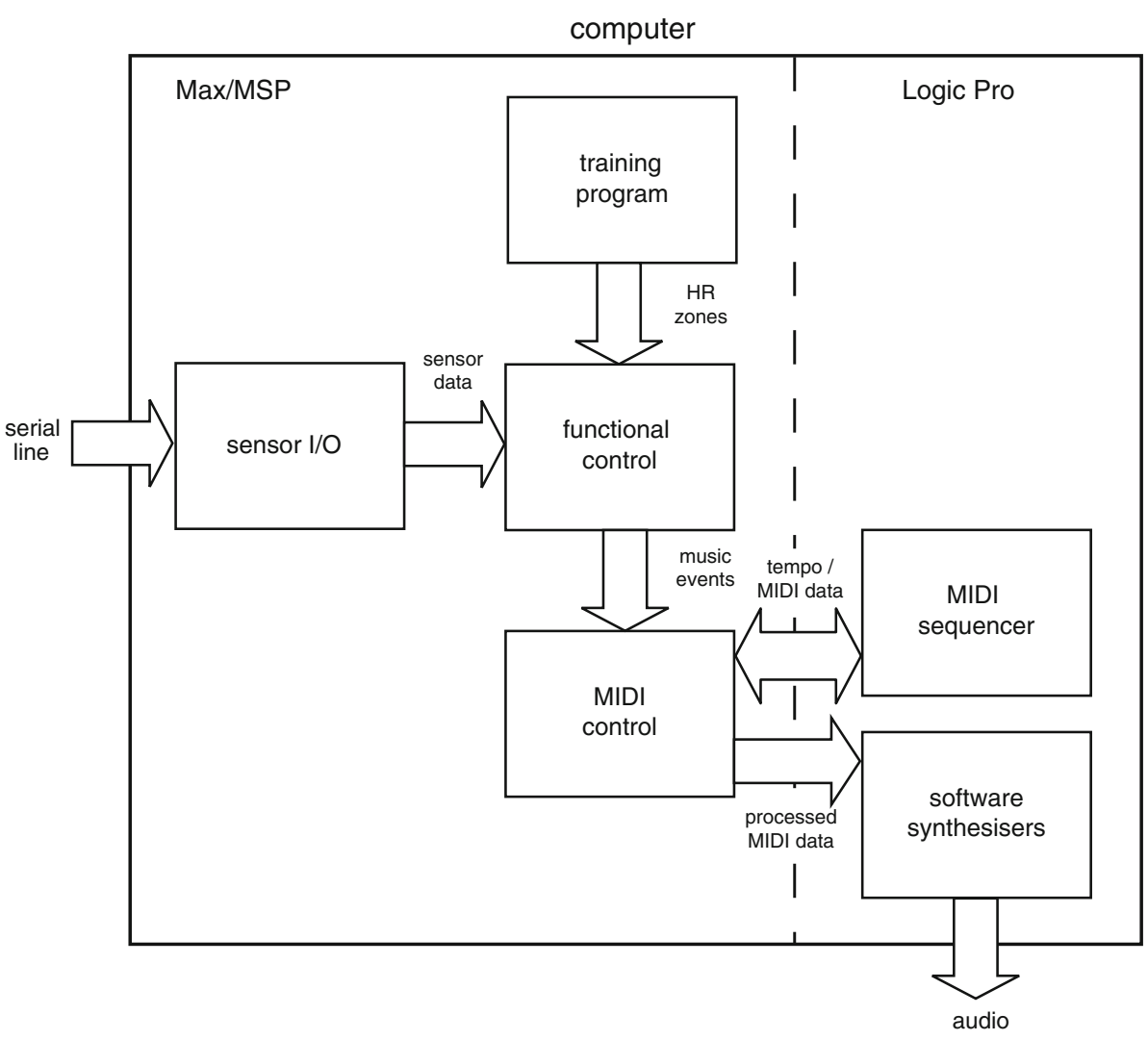

prototype, layer one is the basic rhythm of the music defined by one or more drumbeats. Layer two consists of additional percussion sounds (cymbals and drums). Layer three consists of a base synthesizer and melodic synthesizer notes. The higher the level of compliance, the higher the musical richness.

3. Users are guided in their training intensity by receiving auditory feedback if they either need to speed up or slow down. This happens in two occasions: when the user is outside of the training intensity zone (below the lower or exceeding the upper limit), or when there is an upcoming change in training intensity due to the prescribed training program. In each case, synthesized tones are played (integrated in the music) with a rising pitch to speed up, and with a decreasing pitch to slow down. 


\section{Pilot Investigation}

We conducted a within-subject experiment in which the auditory feedback was the independent variable with two conditions; interactive music and audible alarms. System effectiveness, intrinsic motivation, attentional focus and rate of perceived exertion were the dependent variables. Traditional HR monitors commonly use audible alarms and these were replicated to serve as a reference.

From literature (Karageorghis and Terry 2001; Terry and Karageorghis 2006) we know that music has a motivational effect on exercising. To guide an effective exercise session, monitoring HR and guiding by means of prescribing target heart rate are both essential. To investigate the success of moBeat, which attempts to combine these two factors (music and monitoring), we assessed the influence of both conditions on effectiveness (compliance to the HR zones), intrinsic motivation, attentional focus and rate of perceived exertion (RPE) (as is clarified in the measurement section). Based on the known effect music has on exercise we hypothesized that:

1. Exercising with the music-based moBeat system will lead to a higher intrinsic motivation (assessed with the Intrinsic Motivation Inventory, IMI), which is assumed to be accompanied by increased external focus of attention (measured with the Attentional Focus Questionnaire, AFQ) and lower perceived exertion, as assessed with the perceived exertion (RPE) scale, when compared to an existing system.

2. The effectiveness of the moBeat system will be comparable to the effectiveness of using audible alarms, as is currently the golden standard in HR monitoring.

\section{Participants}

26 Philips employees-5 women and 21 men-participated $^{1}$ in the study. The age ranged from 23 to 51 years, with an average age of 33.7. All 26 subjects completed both exercise sessions successfully without any problems. At least all subjects had some exercise experience and a few of them were sports enthusiasts. Due to NDA (Non Disclosure Agreement) issues, participants were restricted to be Philips employees. ${ }^{2}$ Before participation, subjects agreed with, and signed a written informed consent form.

\footnotetext{
${ }^{1}$ Participants were employees of Philips coming from different departments within Philips and had various backgrounds. They participated voluntarily and did not receive any payment.

2 The project was a cooperation between the Department of Industrial Design and Philips Research Eindhoven. Philips Research is a part of Royal Philips Electronics Inc. and served as an industrial partner, which is common in the Industrial Design discipline due to the
}

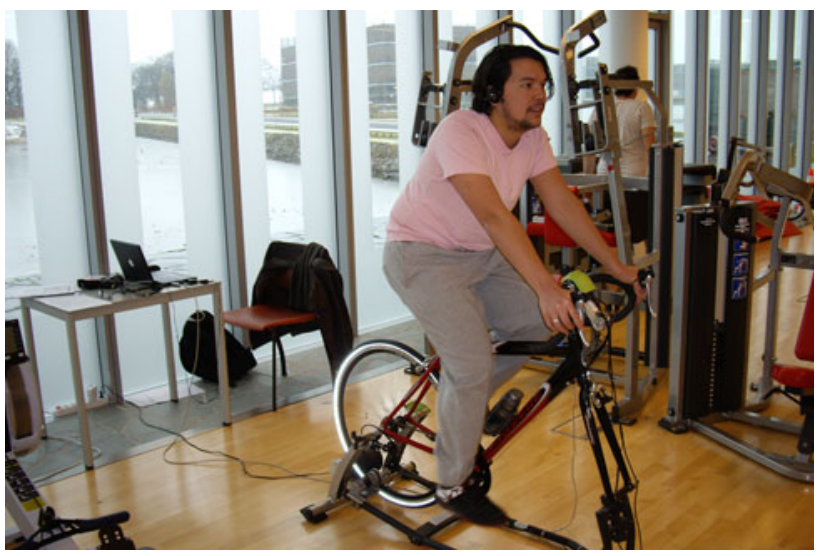

Fig. 4 One of the participants exercising during a test sessions

\section{Procedure}

To benchmark moBeat, we compared it to the audible feedback many of today's HR monitors are capable of providing. These systems generally use both a graphical display and simple audible alarms to indicate noncompliance with the predefined intensity zones. To assure that only the difference in auditory feedback was investigated-instead of audio-visual versus audio-participants were only able to listen to the systems.

During the experiment, participants used the prototype system and the reference system, cycling on a stationary cycle. The sessions took place in the 'Wellness Center' situated on the High Tech Campus Eindhoven. Figure 4 shows one of the participants using the experimental setup. The experiment consisted of two training sessions with a total duration of 20 min each. Each session was divided in three stages; 5 min warming-up, 10 min interval training with 2.5 min intervals, and 5 min cooling-down.

To take physiological differences between the subjects into account, the intensity zones were calculated using the Karvonen method (Karvonen et al. 1957) instead of using absolute values. The Karvonen method (THR $=\left[\left(\mathrm{HR}_{\max }-\right.\right.$ $\left.\mathrm{HR}_{\text {rest }}\right) \times \%$ Intensity) $\left.+\mathrm{HR}_{\text {rest }}\right]$ uses resting heart rate $\left(\mathrm{HR}_{\text {rest }}\right)$ and maximum heart rate to calculate the target heart rate (THR). Example: for someone with an $\mathrm{HR}_{\max }$ of 180 and an $\mathrm{HR}_{\text {rest }}$ of 70 : $50 \%$ intensity: [(180-70) $\times$ $0.50]+70=125$ BPM. $85 \%$ intensity: $[(180-70) \times$ $0.85]+70=163$ BPM. The intensity varied between HRrest to $50 \%$ for the warming-up, and intervals of 60,65 and $70 \% \pm 5$ heartbeats for the exercising stage. For the cooling-down stage, there was no strict heart rate zone because this is not relevant from a physiological

Footnote 2 continued

commercial aspect of the field. Philips Research gave financial support for the development of the prototype and provided the necessary equipment. 
perspective. Keeping the heart rate below the lower limit of the previous interval was rewarded by means of auditory feedback. With the help of a physiologist, we chose these values to physically challenge the subjects to simulate a realistic training session without exhausting them. $\mathrm{HR}_{\text {max }}$ was calculated for each subject using the age-related formula: $\mathrm{HR}_{\max }=205.8-(0.685 \times$ age $)$. Research (Robergs and Landwehr 2002) on 43 different formulas for predicting $\mathrm{HR}_{\max }$ (including the one above) concluded that this is the most appropriate formula. This was found to have a Standard Deviation of 6.4 BPM and was still considered to be acceptable for the use of prescribing exercise intensities. The subjects were asked to measure their resting heart rate in advance of the experiment. If the participants failed to do so, they were asked to sit and relax before the exercise and their resting heart rate was measured.

The two sessions took place on different days to eliminate possible fatigue due to earlier exercise. The order of the different exercises was counter-balanced. Exercise data were logged automatically. Additionally, after the exercise session subjects were asked to fill in questionnaires to obtain data for intrinsic motivation, attentional focus and rate of perceived exertion, supported by an informal evaluation.

\section{Stimuli}

\section{moBeat}

Compliance to the prescribed zone was rewarded with richer music in 3 steps:

Out of training zone

In training zone

In training zone $>10 \mathrm{sec}$

layer 1: base rhythm

layer 2: base rhythm + additional percussion

layer 3: base rhythm + additional percussion + bass synthesizers and melody

When users were training outside the prescribed zone, they heard either: four notes with increasing pitch to indicate that the current intensity was too low and they needed to speed up, or four notes with decreasing pitch to indicate that the intensity was too high and they needed to slow down. To indicate an upcoming change in training zone, the system played the four notes with either increasing or decreasing pitch, depending on the next zone being at a higher or lower intensity.

\section{Reference System}

The two leading brands for heart rate monitors are Polar and Suunto, both in the form of wrist-top computers (watches) that provide the same fitness functionality as moBeat does. All HR watches are able to give audible feedback in the form of watch-like beep sounds. Each of the brands utilizes its own way of providing feedback.

Most Polar HR watches beep when exceeding the upper or surpassing the lower limit at every measured heartbeat. This results in a slow beeping sound below the lower limit and a fast beeping sound when exceeding the upper limit. Although there is a small difference in interval time between the beeps between both cases, this might be hard to perceive during exercising, especially when the HR zones are relatively narrow (10 BPM in our case).

Suunto uses high-frequency beeping sounds to mark the upper limit and low-frequency beeps to mark the lower limit. These different signals are much easier to discriminate between when using the system with audio only, which is why we selected this system as a reference system for our user experiment. For the audible alarm condition, we replicated the audible alarms produced by an HR watch and used these in our own software; it provides audible feedback on performance by indicating whether the user is training below or above the prescribed zone limit, and by indicating when the intensity zones change.

- . - . - - High-frequency beeps $(150 \mathrm{~Hz})$ to indicate that the user is exceeding the upper limit

- $\quad$ - $\quad$ - $\quad$ Low-frequency beeps $(75 \mathrm{~Hz})$ to indicate that the user is below the lower limit

In order to use the HR monitors in interval training (as needed for the experiment), multiple intervals with different intensities can be set. To warn the user when the interval changes, timers can be set. Usually these are timers, counting down from ten to zero at the end of the interval. To indicate an upcoming change in training zone, the reference system counts down, beeping at 10, 5, 3, 2 and 1 seconds before zone change, and sounding an alarm (- . - . - - ) when the zone change occurs.

\section{Measurements}

\section{Intrinsic Motivation Inventory}

The participant's intrinsic motivation was assessed using the Intrinsic Motivation Inventory (IMI) developed at the University of Rochester ("Intrinsic Motivation Inventory" 2008). The psychometric properties of the IMI questionnaire was assessed in McAuley et al. (1989), who found strong support for its validity and it was successfully used in comparable studies (IJsselsteijn et al. 2004). It comprises the subscales: interest/enjoyment, perceived competence, effort, value/usefulness, felt pressure and tension, and perceived choice when engaging in an activity. We selected three of the subscales based on their relevance to the study as well as their relevance to this specific activity. The 
interest/enjoyment subscale provides a self-report measure of intrinsic motivation. The perceived choice and perceived competence subscales are theorized to be positive predictors of intrinsic motivation as these are important motivational factors. The value/usefulness subscale is used to assess if people internalize and become self-regulating for activities that they consider useful for themselves, which could lead to a higher long-term motivation. We added the usefulness/value subscale to assess the value people assign to the exercise that might lead to a higher motivation (also in the long term). In total, the questionnaire we used in the study had 20 items: interest/enjoyment (7 items), perceived competence (6 items) and value/usefulness (7 items). Respondents were asked to rate each item using a 7-point Likert scale, based on how true the statements were for them.

\section{Attentional Focus Questionnaire}

The Attentional Focus Questionnaire (AFQ) is designed to measure attentional focus directly rather than just to assume that an external focus is being induced. The AFQ has been used extensively in association and dissociation literature, Lind et al. (2009) discuss the psychometric properties of attentional focus. The AFQ (Brewer et al. 1996) is a 30-item questionnaire assessing focus of attention during endurance exercising (originally designed for an endurance run). It contains three subscales: association (11 items), dissociation (12 items), and distress (7 items). Respondents were asked to rate each item using a 7-point Likert scale, with 1 being "I did not do this at all" and 7 being "I did this all the time"). Items on the association scale include "monitoring your pace," "monitoring specific body sensations (e.g., leg tension, breathing rate)", and "paying attention to your rhythm." Examples of items on the dissociation scale are "singing a song in your head," "writing a letter or paper in your head," and "thinking about pleasant images." Items on the distress scale include "focusing on how much you are suffering," "wishing the run would end", and "thinking about how much you want to quit." Some of the items have been slightly altered to make a better fit for the activity performed in this study (e.g. run/running was changed in exercise/cycling).

\section{Rate of Perceived Exertion (RPE)}

RPE, also called the Borg scale (named after its inventor Gunnar Borg) (Borg 1970) is a self-report scale of perceived exercise intensity. It is a well-established scale that has been widely used for assessing perceived exertion both in medicine and sports training and is considered to have a high reliability and validity (Skinner et al. 1973). The scale is a linear scale ranging from 6 to 20 , starting with 6 "nothing at all' and ending with 20 "exhaustion".

Data Logging and Analysis

During the experiment the following data were recorded automatically: time in the correct intensity zone and total time; number of times out of the zone; time of interval change; heart rate; RPM and rear-wheel speed. The total time (in seconds) of heart rate zone compliance was compared between the two systems as well as the number of times (count) subjects got out of the zone. The data obtained from the questionnaires were processed by calculating the mean scores of each subscale. A $p$ value of 0.05 was used as the statistical threshold to indicate statistical significance.

\section{Results}

\section{System Effectiveness Comparison}

The results of the 26 sessions show no significant difference in effectiveness between the systems. We conducted a repeated measure analysis of variance (ANOVA) in which the system (interactive music versus audible alarms) was the within subject factor and compliance and times out-of-zone were the measurements. It revealed that our moBeat system had no significant influence on compliance $(\mathrm{F}(1,25)=$ $2.024, p=0.167)$ and times out-of-zone $(\mathrm{F}(1,25)=1.514$, $p=0.230$ ). Figure 5 shows the mean values of compliance (number of seconds they spent in the target HR zone) of all 26 participants in both conditions.

\section{Questionnaires}

We conducted a reliability analyses on the IMI and AFQ scales. The Cronbach's alpha was 0.940 for the IMI and

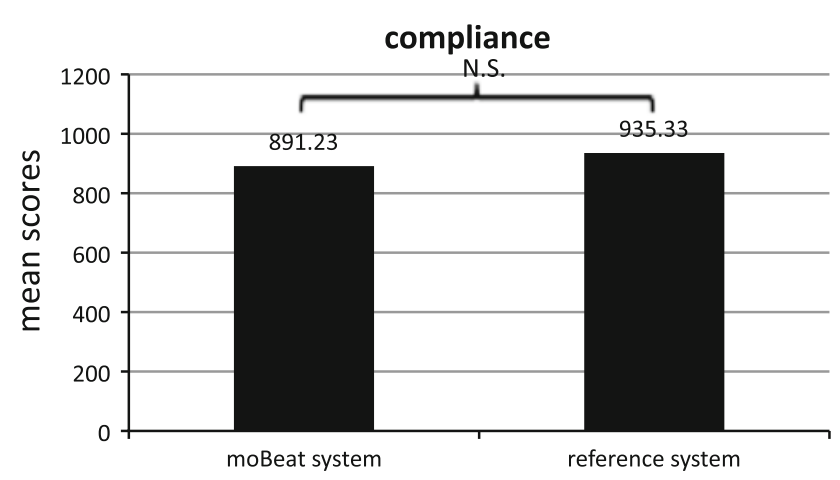

Fig. 5 Graph displaying mean values for compliance 
0.752 for the AFQ. According to Nunnally (1978), these values are well above the threshold of 0.70 .

Results from the Intrinsic Motivation Inventory (IMI) and Attentional Focus Questionnaire (AFQ) showed important differences. We again conducted a repeated measure analysis of variance (ANOVA) in which the system (interactive music versus audible alarms) was the within subject factor, and IMI subscales fun/enjoyment, perceived competence, value/usefulness, AFQ subscales dissociation, association, distress and the Borg scale (RPE) were the measurements. The system had a significant effect on fun/enjoyment $(\mathrm{F}(1,25)=99.435, p<0.001)$, perceived competence $(\mathrm{F}(1,25)=4.239, p=0.05)$, value/ usefulness $(\mathrm{F}(1,25)=5.368, p=0.029)$, dissociation $(\mathrm{F}(1,25)=9.372, \quad p=0.005) \quad$ and distress $(\mathrm{F}(1$, $25)=26.868, p<0.001)$. The system had no significant influence on association $(\mathrm{F}(1,25)=0.162, p=0.691)$ and perceived exertion $(\mathrm{F}(1,25)=1.294, p=0.266)$. Mean values of the RPE scale and IMI and AFQ subscale scores are shown in Figs 6 and 7.

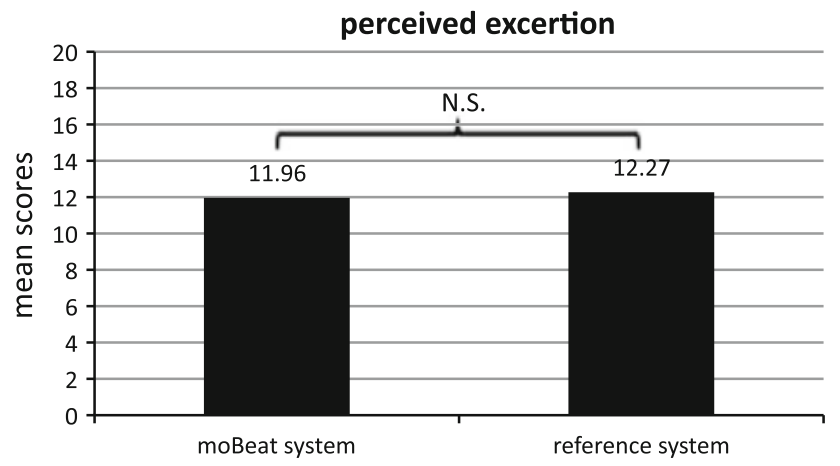

Fig. 6 Graph showing the mean values for perceived exertion

\section{Discussion}

The results showed no significant difference in compliance and times out-of-zone between our moBeat system and the reference system, indicating that both systems were comparable with regard to effectiveness. A higher score on the fun/ enjoyment subscale indicates a higher measure of intrinsic motivation in the interactive music condition with our moBeat system. This is backed by a higher score on the perceived competence scale, which is considered a positive predictor of intrinsic motivation. A higher score on the value/usefulness scale can be considered as a positive property of a system, as people internalize and become self-regulating with respect to activities that they experience as useful or valuable for themselves ("Intrinsic Motivation Inventory" 2008).

On the AFQ subscales dissociation and distress; the subjects scored significantly lower in the condition with interactive music while the level of association remained more or less equal. Despite the fact that the association remained the same (in both conditions they focused equally on the exercise itself), they engaged more in dissociative strategies in the condition with the reference system. This indicates that musical coaching with interactive music causes natural distraction, away from the exercise itself. A lower score on the distress scale indicates a lower focus on physical pain and discomfort in the interactive music condition. These differences are to be considered advantages of moBeat over its competing heart-rate-based guidance systems. Despite a lower score of moBeat on the perceived exertion scale, (mean values) no significant difference was found between the systems, in contrast with the findings of (Karageorghis and Terry 2001), who stated that music can lead to lower RPE ratings.
Fig. 7 Graph showing the mean values of questionnaire scores for IMI and AFQ

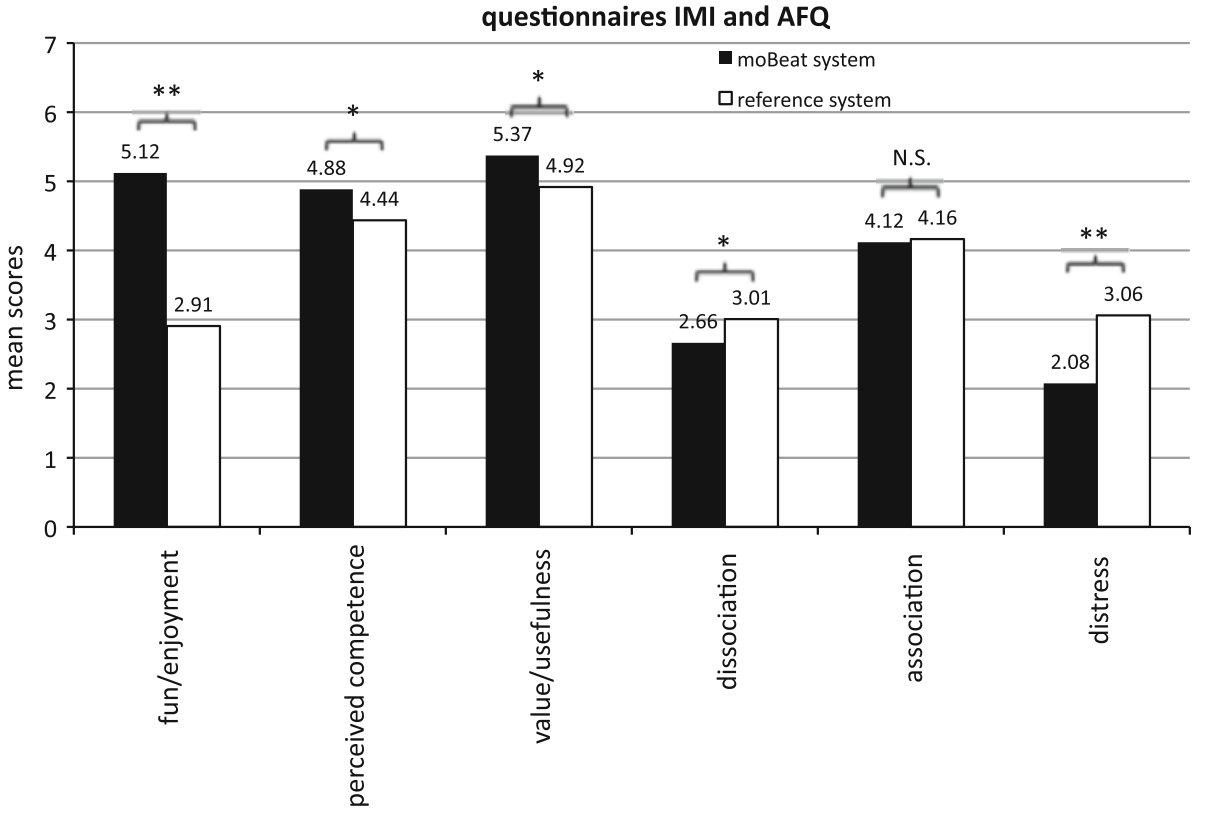




\section{Conclusions}

From the results we conclude that our moBeat system provides higher intrinsic motivation. Users engage less in dissociative strategies and focus less on distress cues. However, the perceived exertion is not significantly lower with moBeat. Still, the moBeat system is as effective as the reference system, which is currently the golden standard in exercise guiding. Informal evaluations after the test sessions revealed that moBeat was more pleasant to use and was as easy to use as the reference system.

We confirmed the results of Terry and Karageorghis (2006) that music in an exercise context has a motivational effect, distracts from unpleasant feelings and suppresses boredom. Additionally, feedback on training pace by means of music tempo seemed to positively influence the consistency in cycling pace.

\section{Limitations}

Using company employees as test participants, however, inevitable at the time due to the NDA, might have biased the results. To limit this, we did not inform the participants about the nature of the research until after finishing both sessions. Further, they were not informed which of the two stimuli was the one being developed.

We cannot make any firm conclusions about long-term motivation of the participants, however, higher scores on intrinsic motivation and a reduced focus on physical pain during the exercise are in favour of improved long-term motivation.

\section{Future Work}

Future work includes system improvements and more rigorous evaluations over extended time periods.

Currently a personal computer is needed to run the software. In future applications, the PC will preferably be replaced with a wearable hardware device like a smart phone, PDA or MP3-player-like device or a dedicated wearable hardware device.

From the observations and informal evaluations after the experiments, we found potential bottlenecks and points for improvements. They include enriching the feedback and providing more precise feedback to the user about how well the exercise is progressing. The music used in the current prototype needs to be improved. In future versions, musical layers would preferably be in other widely available formats such as MP3, and time-stretching algorithms could be used to perform time stretching without adjusting the pitch. Layering MP3 music could be done beforehand; algorithms might process the users' own music database to make it suitable for exercise use to assure users can have their own favourite music. Another approach would be to remain with MIDI or develop a similar format and to invite artists to compose custom-made tracks for exercising purposes.

The system is currently set-up for use on a stationary bicycle in the home environment. Due to the intended portability of the system, it would also be very suitable for outdoor sports like outdoor running and cycling.

Acknowledgments We would like to thank: people from the MCBL project team and the DSP department (Philips Research) for their help and input, Mathias Funk (TU/e) for his help and feedback on developing the music/prototype and all the participants of the experiment for their time, effort and constructive feedback.

Open Access This article is distributed under the terms of the Creative Commons Attribution Noncommercial License which permits any noncommercial use, distribution, and reproduction in any medium, provided the original author(s) and source are credited.

\section{References}

American College of Sports Medicine. (2006). ACSM's guidelines for exercise testing and prescription (7th ed.). Philadelphia: Lippincott Williams and Wilkins.

Apple Inc. (2008). Logic Pro 8 (Version 8.0.2) [Software]. Available from http://www.apple.com/logicstudio/logicpro/.

Borg, G. (1970). Perceived exertion as an indicator of somatic stress. Scandinavian Journal of Rehabilitation Medicine, 2(2), 92-98.

Brewer, B. W., Van Raalte, J. L., \& Linder, D. E. (1996). Attentional focus and endurance performance. Applied Research in Coaching and Athletics Annual, 11, 1-14.

Cycling '74. (2008). Max 5 (version 5) [software]. Available from http://www.cycling74.com/products/max5.

Deci, E. L., \& Ryan, R. M. (1985). Intrinsic motivation and selfdetermination in human behavior. New York, NY: Plenum Press.

IJsselsteijn, W., de Kort, Y., Westerink, J., de Jager, M., \& Bonants, R. (2004). Fun and sports: Enhancing the home fitness experience. Paper presented at the international conference on entertainment computing.

Intrinsic Motivation Inventory. (2008). Retrieved 30 Nov 2008, from http://www.psych.rochester.edu/SDT/measures/word/IMIfull. doc.

Nike+iPod. (2008). Nike+iPod. Retrieved 26 Dec 2008, from http://nikeplus.nike.com/nikeplus/.

Jette, M., Campbell, J., Mongeon, J., \& Routhier, R. (1976). The Canadian home fitness test as a predictor of aerobic capacity. Canadian Medical Association Journal, 114(34), 680-682.

Julio, D. (2008). Heart rate monitor interface user manual. Retrieved 1 Oct 2008, from http://danjuliodesigns.com/sparkfun/hrmi_ assets/hrmi.pdf.

Karageorghis, C. I., \& Terry, P. C. (1997). The psychophysical effects of music in sport and exercise: A review. Journal of Sport Behavior, 20, 54-68.

Karageorghis, C. I., \& Terry, P. C. (2001). The magic of music in movement. Sport and Medicine Today, 5, 38-41.

Karageorghis, C. I., Terry, P. C., \& Lane, A. M. (1999). Development and validation of an instrument to assess the motivational qualities of music in exercise and sport: The Brunel music rating inventory. Journal of Sports Sciences, 17, 713-724. 
Karvonen, M. J., Kentala, E., \& Mustala, O. (1957). The effects of training heart rate: A longitudinal study. New Annals Medicinae Experimentalis et Biologiae Fenniae, 35(3), 307-315.

Lind, E., Welch, A. S., \& Ekkekakis, P. (2009). Do 'mind over muscle' strategies work? Examining the effects of attentional association and dissociation on exertional, affective and physiological responses to exercise. Sports Medicine, 39(9), 743-764.

McAuley, E., Duncan, T., \& Tammen, V. V. (1989). Psychometric properties of the intrinsic motivation inventory in a competitive sport setting: A confirmatory factor analysis. Research Quarterly for Exercise and Sport, 60, 48-58.

MiCoach. (2008). Adidas miCoach. Retrieved 26 Dec 2008, from http://www.micoach.com/.

Nintendo. (2008). Wii. Retrieved 26 Dec 2008, from http://www.nin tendo.co.uk/NOE/en_GB/wii_54.html.

Nunnally, J. C. (1978). Psychometric theory (2nd ed.). New York: McGraw-Hill.

Polar Electro. (2008). Polar. Retrieved 26 Dec 2008, from http:// www.polar.fi.

Robergs, R. A., \& Landwehr, R. (2002). The Surprising history of the "HRmax $=220$-age" equation. Journal of exercise physiology, $5,1-10$.
Ryan, R. M., Frederick, C. M., Lepes, D., Rubio, N., \& Sheldon, K. M. (1997). Intrinsic motivation and exercise adherence. International Journal of Sport Psychology, 28, 335-354.

Skinner, J. S., Hutsler, E., Bergsteinova, V., \& Buskirk, E. R. (1973). The validity and reliability of a rating scale of perceived exertion. Medicine and Science in Sports, 5(2), 94-96.

Suunto. (2008). Suunto training world. Retrieved 26 Dec 2008, from http://www.suunto.com/suunto/Worlds/main/index.jsp.

Szabo, A., Small, A., \& Leigh, M. (2001). The effects of slow- and fast-rhythm classical music on progressive cycling to voluntary physical exhaustion. Journal of Sports Medicine and Physical Fitness, 39(3), 220-225.

Terry, P. C., \& Karageorghis, C. I. (2006). Psychophysical effects of music in sport and exercise: An update on theory, research and application. Paper presented at the joint conference of the Australian psychological society and the New Zealand psychological society.

Wijnalda, G., Pauws, S., Vignoli, F., \& Stuckenschmidt, H. (2005). A personalized music system for motivation in sport performance. IEEE Pervasive Computing, 4(3), 26-32.

Yamaha. (2008). BODiBEAT. Retrieved 12 June 2008, from http:// www.yamaha.com/bodibeat/. 